\title{
Predictability, bred vectors, and generation of ensembles in space-time chaotic systems
}

\author{
Cristina Primo, Miguel A. Rodríguez, Juan M. López, and Ivan Szendro* \\ Instituto de Fisica de Cantabria, Avenida los Castros, 39008 Santander, Spain
}

(Received 9 August 2004; published 18 July 2005)

\begin{abstract}
Spatial configuration of initial errors strongly affects predictability of space-time chaotic systems. The predictability of numerical models can be adjusted by using prepared ensembles of initial conditions. We present a natural way of preparing ensembles based in using finite-amplitude perturbations with varying correlation. This allows one to take into account the underlying dynamics to generate initial perturbations with spatial correlations varying from fully correlated (bred vectors) to random fluctuations.
\end{abstract}

DOI: 10.1103/PhysRevE.72.015201

PACS number(s): 05.45.Jn, 05.45.Ra, 89.75.Da, 92.60.Wc

Early predictability questions were formulated in the field of weather forecasting by Lorenz [1], giving rise to the renaissance of the science of chaos. The Lorenz model is a simple representation of atmospheric evolution that exhibits low-dimensional chaos. It is well known that prediction time of low-dimensional chaotic systems can be estimated as the inverse of the Lyapunov exponent (with a logarithmic correction due to the strength of initial errors). Realistic models of atmospheric evolution used in current weather forecasts are spatiotemporal chaotic systems that introduce other ingredients [2]. Important changes concerning predictability can appear from the fact that here errors propagate in space [3]. This process occurs slowly and produces correlated and localized perturbations. Changes in the way we deal with predictable tasks in extended chaotic systems are necessary [4]. Logarithmic sensitivity to initial conditions might not describe predictability if these functions are correlated.

Modern forecasters have realized that prediction now requires the use of ensembles of forecasts, instead of single deterministic ones [5]. A model is predictive when the actual experimental trajectory evolves like a member of the ensemble and the range of prediction coincides with the saturation time of ensemble fluctuations. The crucial point is that, with the same initial variance, the growth of initial fluctuations depends on the spatial form of ensembles. This fact allows one to prepare conveniently ensembles of initial conditions. But calibration of ensembles in a given model is usually a phenomenological task. The use of complicated models does not allow systematic analysis and little is known about implications of spatial chaos on predictability. Our point of view in this paper is totally different. We deal with simple models of space chaos trying to extract general results valid for other systems. The methods used in weather forecasting will be the guide in this study: We try to explain some results obtained phenomenologically in this field and suggest different methods that emerge from our analysis. In a perfect model case finite fluctuations evolve as true errors; they become progressively correlated during evolution close to the tangent space and uncorrelated when arriving to a given amplitude error, farther from the tangent space [6]. The use of these correlated fluctuations as ensembles of initial

*Electronic address: rodrigma@ifca.unican.es conditions can lead to a systematic method of calibration, since the saturation time of fluctuations strongly depends on initial correlation. In weather forecasting, totally correlated finite fluctuations are widely used as initial conditions. They are called bred vectors (BVs). BVs are stationary finite fluctuations that are periodically renormalized to prevent exponential growth. They were used for operative forecasting in the National Center for Environmental Prediction (NCEP) $[7,8]$. Their use has several advantages. On the one hand, they provide a certain kinetic memory of the spatial distribution of errors. On the other, they can be computed from the original nonlinear model without the corresponding linearized counterpart. Therefore, breeding techniques constitute an important tool in modern weather forecasting, both for operative uses [9] and for research [10]. But important questions concerning statistical and dynamical properties of BVs in space-time chaotic systems are still unanswered and some of them are of practical interest. Standard BVs are totally correlated finite fluctuations, and when used as initial conditions they have too long a saturation time. This is a weakness of the method that limits its application to cases with small model errors. In fact several artifices, either adding noise externally, manipulating amplitudes [11], or orthogonalizing [12] vectors are used to achieve more uncorrelated BVs. In this paper we show how to obtain stationary finite fluctuations with controlled correlation using an alternate breeding method. We assume the hypothesis of the perfect model with homogeneous errors, focusing our research on the prediction of global variables.

Our results are based on numerical simulations of coupled map lattices in one dimension. They are simple model systems exhibiting space-time chaos. We consider a diffusively coupled map array of $L$ chaotic oscillators with dynamics given by

$$
\begin{aligned}
u(x, t+1)= & \nu f(u(x+1, t))+\nu f(u(x-1, t)) \\
& +(1-2 \nu) f(u(x, t))
\end{aligned}
$$

where $x=1,2, \ldots, L, f(u)$ is a chaotic map, $\nu$ is the coupling constant, and periodic boundary conditions are imposed. The coupling parameter has been fixed at $\nu=1 / 3$ in all the simulations presented in this paper. We have carried out simulations for several choices of the map, in one and two dimensions and also for other chaotic oscillators, as, for instance, 
the Lorenz one, obtaining the same general results. For the sake of brevity, all the simulations we present below correspond to the coupled logistic maps, $f(u)=4 u(1-u), 0 \leqslant u$ $\leqslant 1$. Perturbations are obtained by considering the evolution of trajectories whose initial conditions are close to a reference (or control) trajectory. This reference trajectory is the solution $u^{0}(x, t)$ of Eq. (1) with an initial condition $u^{0}(x, 0)$. Under the perfect model approximation the control trajectory represents the experimental evolution. An ensemble of finite perturbations $\{\delta u(x, t)\}$ is obtained by taking the difference $\delta u(x, t)=u(x, t)-u^{0}(x, t)$ between the control trajectory and every one of the disturbed solutions. These are generated by iterating (1) for an ensemble of initial conditions $u(x, 0)$ $=u^{0}(x, 0)+\delta u(x, 0)$. Since we are interested in the propagation of real finite errors, linearization of (1) has been avoided. Moreover, to improve the statistical characterization of global variables averages over both, initial conditions and different control trajectories are performed.

Logarithmic-normal infinitesimal fluctuations. For the statistical characterization of infinitesimal fluctuations, it is convenient to use a picture presented by Pikovsky and Politi [13]. There it is shown that the logarithmic transformation of infinitesimal fluctuations $l_{\delta u}(x, t)=\ln (|\delta u(x, t)|)$ evolves as a rough interface in the Kardar-Parisi-Zhang (KPZ) universality class, with well-known statistics and scaling laws. The statistics of KPZ rough interfaces is known to be Gaussian in the stationary state. This suggests that infinitesimal errors, taken as absolute values of fluctuations, converge to logarithmic-normal distributions. The logarithmic-normal character of error growth has already been shown in lowdimensional chaotic systems [14]. The same behavior is expected in extended systems since it is simply an effect of the exponential growth. Standard methods of analysis, better adapted to deal with Gaussian processes, could be not longer suitable. In fact, the logarithmic-normal distribution is characterized by the mean and variance of the logarithmic transformation, which suggests the use of geometrical means in the field of fluctuations. So we use the amplitude of fluctuations defined by $\epsilon_{\delta u}(t)=\exp \left[\left\langle\overline{l_{\delta u}(x, t)}\right\rangle\right]$ and the width of the interface $w_{\delta u}(t)=\left\langle\overline{\left\langle l_{\delta u}(x, t)-\left\langle\overline{l_{\delta u}}\right\rangle\right]^{2}}\right\rangle^{1 / 2}$ for a proper characterization of fluctuations. The overbar stands for spatial mean while $\langle\cdots\rangle$ means the ensemble average. Arithmetical means in the logarithmic transformed field $l_{\delta u}$ translate into geometrical ones in the field of fluctuations $\delta u$.

Saturation time. The behavior of finite-size fluctuations in terms of these magnitudes has been studied by us in [6]. We have shown that the amplitude grows exponentially in time $\epsilon_{\delta u}(t) \sim \epsilon_{\delta u}(0) \exp (\lambda t)$, until the saturation that occurs, independently of initial conditions, when reaching a given threshold $\epsilon_{n l}, \epsilon_{\delta u}\left(t_{s}\right) \sim \epsilon_{n l} . \lambda$, as shown in [6] and [13], is simultaneously the interface velocity and the leading Liapunov exponent. Saturation of the amplitude occurs at $t_{s}$ $\sim(1 / \lambda) \ln \left[\epsilon_{n l} / \epsilon_{\delta u}(0)\right]$, simultaneously when fluctuations become uncorrelated. This saturation time is used in forecasting as a first index to calibrate ensembles and as a measure of the range of predictability [2]. In a perfect model case, and with well-defined initial errors, this is the prediction time.

Correlations. Finite fluctuations become progressively correlated while evolving as infinitesimal fluctuations close to the tangent space [6]. Correlation lengths can then be used as a measure of the memory of the underlying dynamics carried by fluctuations. But correlation lengths are not easily computed in the field of fluctuations. In general these difficulties are present in the calculation of every other quantity of a logarithmic-normal distribution and can be avoided by using the logarithmic-transformed field. Here it is possible to use the dynamical roughening picture in which the correlation length is straightforwardly defined and evolves as a power law $l_{c}(t) \sim t^{1 / z}$ with universal exponents $\left(z=\frac{3}{2}\right.$ in the KPZ class). Invoking again this scaling, we can measure correlation lengths by means of the already defined width $w$ since both are related by $w \sim l_{c}^{\alpha}, \alpha$ being the roughness exponent $(1 / 2$ in the KPZ class). In this way correlation and the memory of dynamics can be easily quantified by the size of $w$.

Why Bred Vectors? BVs have been used as well-adapted ensembles of initial conditions. It has been explained by a phenomenological argument saying that they carry some of the underlying dynamics, and errors are spatially distributed according to this dynamics. More precise arguments can explain these facts by simply assuming the logarithmic character of BVs. For fluctuations $\delta u(x, t)$ and their logarithmic transformation $l_{\delta u}(x, t)$ one has for the variance $\sigma_{\delta u}^{2}=\left\langle\overline{\delta u^{2}}\right\rangle$ $=\epsilon_{\delta u}^{2} \exp \left(2 \theta_{\delta u}^{2}\right)$, where $\theta_{\delta u}$ is defined in terms of cumulants $\left\langle\left\langle l_{\delta u}\right\rangle\right\rangle$ as $\theta_{\delta u}^{2}=\sum_{r=2}^{\infty} 2^{r-1}\left(\left\langle\left\langle l_{\delta u}\right\rangle\right\rangle / r\right.$ !) $\quad$ [6]. In the case of logarithmic-normal distributions, cumulants of order greater than 2 vanish and $\theta_{\delta u}$ becomes the width $w_{\delta u}$, so we can consider $\theta_{\delta u}$ as a generalized width that also accounts for correlation. Hence one obtains the interesting relationship

$$
\sigma_{\delta u}=\epsilon_{\delta u} \exp \left(\theta_{\delta u}^{2}\right),
$$

relating errors (typical deviation) with amplitudes and correlations. It states that for the same level of error $\sigma_{\delta u}$ one has fluctuations that are more or less correlated depending on the amplitude. Hence, a more correlated initial condition will have lower initial amplitude and therefore a longer range of predictability,

$$
t_{s} \sim \frac{1}{\lambda} \ln \left[\frac{\sigma_{\delta u}\left(t_{s}\right)}{\sigma_{\delta}(0)}\right]+\frac{\theta_{\delta u}(0)^{2}-\theta_{\delta u}\left(t_{s}\right)^{2}}{\lambda} .
$$

The first contribution in this equation would correspond to the prediction time obtained with Gaussian random initial conditions, whereas the second one is the gain by correlation. Since breeding produces correlation, this is the gain due to breeding. Note that the maximum gain in prediction time due to breeding is proportional to the system size $L: \max \left(\theta^{2}\right.$ $\left.\sim w^{2}\right) \sim L^{2 \alpha}(\alpha=1 / 2$ in KPZ class $)$ and $\theta_{\delta u}\left(t_{s}\right)=0.302068$ for Gaussian final errors. In this case predictability is governed by the diffusion of errors originated deterministically. Note that an extended chaotic system has a chaos dimension proportional to $L$. Then, paradoxically, the more chaotic a system is, the more predictable it becomes. On the other hand, it now becomes clear that both parameters, deviation of errors, and correlations are independent and necessary to fit correctly dynamically compatible initial conditions. Let us focus now on this point.

Bred vectors with constant norm. Bred vectors were de- 


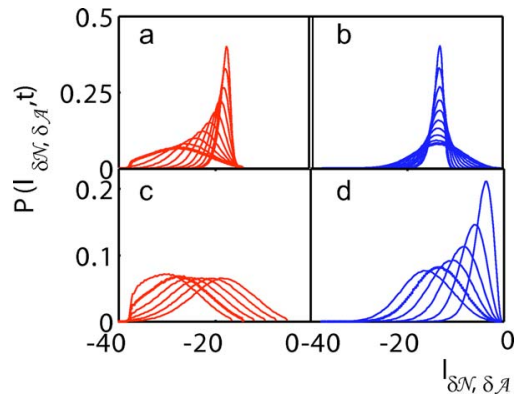

FIG. 1. (Color online) Probability density of the logarithmic transformed BVs. Upper panels: Plotted at time $2^{n}(n=1,2, \ldots, 11)$, (a) with constant norm $\sigma_{0}=10^{-6}$ and (b) with constant amplitude $\epsilon_{0}=10^{-6}$. Lower panels: At the stationary state with constant norm, (c) $\left(\sigma_{0}=10^{-3}, 10^{-4}, \ldots, 10^{-8}\right)$ and constant amplitude, (d) $\left(\epsilon_{0}\right.$ $=10^{-3}, 10^{-4}, \ldots, 10^{-8}$ )

fined in analogy with the operation of data reassimilation in numerical models of atmospheric evolution. The output of the numerical model is corrected by the observed experimental data in short periods of time $\left\{\tau_{1}, \tau_{2}, \tau_{3}, \ldots,\right\}$. Instead of assimilation of data, bred vectors are defined by multiplying each member of the ensemble by a reduction factor $\left\{k_{1}, k_{2}, k_{3}, \ldots\right\}$ maintaining perturbations with a constant size. In essence this is a generalization of the method used to obtain the leading Liapunov vector in low-dimensional chaotic systems adapted to numerical weather models. Rescaling of the norm prevents the exponential growth that allows one to evolve fluctuations for long times without numerical errors. Standard methods used in operative forecasts take uniform intervals of time $\delta \tau$ normalizing to a size defined by some norm. Here we take $\sigma_{0}=\overline{\delta u^{2}} 1 / 2$ as norm. The ensemble of bred vectors are then defined by these two parameters $\delta \tau$ and $\sigma_{0}$. We have reproduced this methodology with our model Eq. (1) taking an ensemble of finite perturbations around a reference trajectory, as pointed out above. Now BVs are constructed by normalizing each evolved perturbation $\left\{\delta u_{i}(x, t)\right\}$ at intervals $\tau_{i}=\tau_{i-1}+\delta \tau$ adjusting the norm in each step to $\sigma_{0}$

$$
\delta \mathcal{N}\left(x, \tau_{i}\right)=\sigma_{0} \frac{\delta u_{i-1}\left(x, \tau_{i}\right)}{\left[\delta u_{i-1}\left(x, \tau_{i}\right)^{2}\right]^{1 / 2}} .
$$

The evolution of finite perturbations $\left\{\delta u_{i}(x, t)\right\}$ within each interval $\left(\tau_{i}, \tau_{i+1}\right)$ is obtained by taking the difference $\delta u_{i}(x, t)=u_{i}(x, t)-u^{0}(x, t)$ between the reference trajectory and every one of the perturbed solutions whose initial condition at time $\tau_{i-1}$ is $u_{i}\left(x, \tau_{i-1}\right)=\delta \mathcal{N}\left(x, \tau_{i-1}\right)+u^{0}\left(x, \tau_{i-1}\right)$. We have two varying parameters $\sigma_{0}$ and $\delta \tau$ to generate BVs but only $\sigma_{0}$ is actually relevant. In fact, $\delta \tau$ only changes the magnitude of the reduction factor as $k \sim \exp (\lambda \delta \tau)$, and here is fixed to one. The definitions used in fluctuations, $l_{\delta u}, w_{\delta u} \ldots$, are generalized to $\mathrm{BVs}, l_{\delta \mathcal{N}}, w_{\delta \mathcal{N}} \ldots$, simply by taking $\delta \mathcal{N}$ instead of $\delta u$. In Fig. 1(a) we plot the probability density of the logarithmic transformed bred vectors, $P\left(l_{\delta \mathcal{N}}, \tau\right)$ at different times. We observe that fluctuations generated during this breeding evolve with a width increasing as in common (not bred) fluctuations $w_{\delta \mathcal{N}}(t) \sim t^{\beta}$ [6], but with an

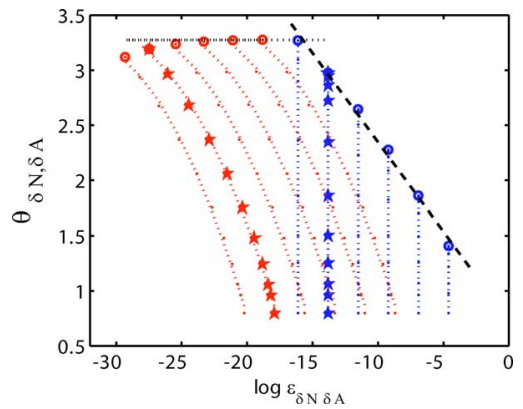

FIG. 2. (Color online) Generalized width $\theta$ vs logarithmic amplitude during breeding: With constant norm (curved lines) and constant amplitude (vertical lines). Symbols represent states plotted in Fig. 1. Nonlinear threshold is depicted by a dashed line and system size saturation width with a dotted one.

amplitude constrained by $\sigma_{0}=\left\langle\overline{\delta \mathcal{N}^{2}}\right\rangle^{1 / 2}=\epsilon_{\delta \mathcal{N}} \exp \left(\theta_{\delta \mathcal{N}}^{2}\right)$. So, as time goes by the mean value of the plotted distribution, which is the logarithmic amplitude $\ln \epsilon_{\delta \mathcal{N}}$, shifts to lower values. We plot the trajectory of this evolution in coordinates $\theta_{\delta \mathcal{N}}$ vs $\ln \epsilon_{\delta \mathcal{N}}$ in Fig. 2. It is perfectly fitted by $\theta_{\delta \mathcal{N}}=\left(\ln \sigma_{0}\right.$ $\left.-\ln \epsilon_{\delta \mathcal{N}}\right)^{1 / 2}$ (dotted red lines) in agreement with our theory. On the other hand we can see clearly in Fig. 2 why fluctuations remain in the tangent space while breeding. As we have already mentioned, fluctuations leave the tangent space when the amplitudes cross a given threshold, plotted in a dashed black line in Fig. 2. If this threshold is not crossed at the initial time it is never crossed during the evolution, since amplitudes shift to lower values. Then BVs become totally correlated when the correlation length equals the size of the system and saturate for $w_{\delta \mathcal{N}} \sim w_{L}$ (dotted black line in Fig. 2 ). By varying the parameter of breeding $\sigma_{0}$ all we can obtain with this method is a change in the amplitude of BVs but not in correlations. This is what can be seen in Fig. 1(c). We show in this figure the stationary probability density of the logarithmic transformed $\mathrm{BVs}$ for several values of $\sigma_{0}$. Also in Fig. 2 we can see how BVs are always totally correlated as if they were Liapunov vectors (infinitesimal fluctuations). It is clear that these kinds of BVs are insufficient to represent properly initial conditions in real situations where models errors are present and less correlated initial conditions could be needed.

Bred vectors with constant amplitude. Observing Fig. 2 one could have easily thought of a breeding method for generating noninfinitesimal fluctuations. It suffices to avoid the shift of amplitude, for instance, by keeping it constant. So, instead of a typical deviation or any other arithmetical mean of fluctuations we take amplitudes for the definition of size in the breeding process. The new BVs are then identically generated but now the reduction factor is proportional to the amplitude $\epsilon_{0}$,

$$
\delta \mathcal{A}\left(x, \tau_{i}\right)=\epsilon_{0} \frac{\delta u_{i-1}\left(x, \tau_{i}\right)}{\prod_{x}\left(\left|\delta u_{i-1}\left(x, \tau_{i}\right)\right|\right)^{1 / L}},
$$

and the initial condition of finite perturbations $\left\{\delta u_{i}(x, t)\right\}$ for each breeding time interval is $u_{i}\left(x, \tau_{i-1}\right)=\delta \mathcal{A}\left(x, \tau_{i-1}\right)$ $+u^{0}\left(x, \tau_{i-1}\right)$. Then we get the evolution given in Fig. 1(b), 
where obviously the mean value of the distribution remains constant with a value $\ln \epsilon_{0}$, and the width of the probability density grows, as before, until saturation. In Fig. 1(d) we present the stationary density with varying amplitudes. For large enough amplitudes, saturation appears when leaving the tangent space, when the maximum fluctuation crosses the nonlinearity threshold (dashed black line) as shown in Fig. 2. So, the final result of the method is to provide bred vectors with different degrees of correlation. We have now true finite fluctuations with controlled correlation. The ability of the breeding method to generate dynamically compatible ensembles seems to be now strongly enhanced.

Conclusions. In spatiotemporal chaotic systems predictability strongly depends on the spatial correlation of initial conditions. Correlated ensembles have more localized errors, which implies a longer prediction time of global variables. The prediction range of numerical models can then be ad- justed. This can be done naturally by using finite fluctuations controlled by the appropriate breeding process. Nonlinear effects, which are present in finite fluctuations, act only when bred vectors are normalized by amplitudes. In the standard method, using norms, these effects are irrelevant. Nonlinear effects act introducing a threshold for fluctuations, which produces saturation of the growth, leading to partially correlated fluctuations. By varying the amplitude of breeding we can obtain a variation of this scale of correlation to any possible extent. Hence a method to obtain ensembles with varying amplitude and correlation by means of breeding techniques is now feasible. The correlations are generated by the model itself without necessity of external mechanisms. This must be of great interest in applications to predictive methods, such as weather forecasting, since it allows the calibration of any initial condition as a true finite fluctuation.
[1] E. N. Lorenz, J. Atmos. Sci. 20, 130 (1963).

[2] E. Kalney Atmospheric Modeling, Data Assimilation and Predictability (Cambridge University Press, Cambridge 2002).

[3] G. Boffetta, M. Cencini, M. Falcioni, and A. Vulpiani, Phys. Rep. 356, 367 (2002).

[4] T. Bohr, M. H. Jensen, G. Paladin, and A. Vulpiani, Dynamical Systems Approach to Turbulence (Cambridge University Press, Cambridge, 1998).

[5] E. S. Epstein, Tellus 21, 739 (1969).

[6] J. M. López, C. Primo, M. A. Rodríguez, and I. Szendro, Phys. Rev. E 70, 056224 (2004).
[7] M. S. Tracton and E. Kalnay, Weather Forecast. 8, 379 (1993).

[8] Z. Toth and E. Kalnay, Bull. Am. Meteorol. Soc. 74, 2317 (1993).

[9] F. R. Molteni et al., Q. J. R. Meteorol. Soc. 122, 73 (1996).

[10] D. J. Patil et al., Phys. Rev. Lett. 86, 5878 (2001).

[11] T. N. Palmer et al., Ensemble Prediction, Proceedings of the ECMWF Seminar on Validation of Models over Europe (ECMWF, Reading, UK, 1992), Vol. 1.

[12] J. D. Annan, Mon. Weather Rev. 132, 843 (2004).

[13] A. Pikovsky and A. Politi, Nonlinearity 11, 1049 (1998).

[14] R. Benzi and G. F. Carnevale, J. Atmos. Sci. 46, 3595 (1989). 